\title{
HANDSET ANTENNA DESIGN FOR FM RECEPTION
}

\author{
Jaume Anguera $^{*(1,2)}$, David Aguilar ${ }^{(2)}$, Jordi Vergés $^{(2)}$, Miquel Ribó $^{(2)}$, Carles Puente $^{(1)}$ \\ (1) Technology and Intellectual Property Rights Dept. Fractus, Barcelona - Spain \\ (2) Communications and Signal Theory Department \\ Universitat Ramon Llull, Barcelona - Spain \\ Jaume.anguera@fractus.com
}

\begin{abstract}
A passive internal handset antenna for FM reception is presented. Several prototypes have been simulated and tested showing the interesting potential of this solution to eliminate the long cable 1 meter length used at the present time in some mobiles phones.
\end{abstract}

\section{Keywords- Handset antennas, FM reception, small antennas}

\section{INTRODUCTION}

Since the very beginning mobile communications have experimented a continuous growth: new and more sophisticated phones featuring the well-know services such as for example CDMA, GSM, DCS, PCS, and UMTS up to more recent ones such as DVB-H (television). Scientific literature reflects a vast effort in order to find new antenna techniques concerning multi-band and small antennas that need to fit into a very small volume.

A new challenge is appearing on scene: the possibility of receiving FM $(100 \mathrm{MHz}$ central frequency, most common frequency range goes from $88 \mathrm{MHz}$ to $108 \mathrm{MHz}, 20 \%$ bandwidth(BW)) in a mobile phone using an internal antenna, that is, an antenna which is integrated into the volume of a handset phone. The actual approach uses an external antenna consisting of a $1 \mathrm{~m}$. wire approximately. This wire acts twofold: on one hand behaves as a quarter wave monopole at FM frequency and on the other hand carries the demodulated audio signal from the mobile receiver to the microphone placed at the end of such a wire. Therefore, the objective of the present investigation is to show the research carried out: to substitute the external long antenna by a small internal antenna. As a result, the system becomes totally wireless: the signal received by the internal antenna may be sent to a headset using a Bluetooth connection for example.

The paper is divided as follows: a background section where some fundamentals and previous problems are discussed. Section results show the main features of the proposal. Finally, section conclusion summarized the work.

\section{BACKGROUND}

To have an order of magnitude of how small an internal FM antenna is, let us assume the following: consider a typical bar-phone having a total area of $100 \mathrm{~mm} \times 40 \mathrm{~mm}$; antenna should fit within this volume having a low profile. The radiation, BW, and in general, all electromagnetic parameters do not only depend on the radiator itself but of the whole structure [1-3]. In this sense, we can consider as a first approach that the largest diameter of the antenna structure is approximately $107.7 \mathrm{~mm}$ (the diameter matches the handset diagonal as we consider a low profile antenna) which corresponds to $0.0358 \lambda @ 100 \mathrm{MHz}$, resulting in ka=0.11 ( $\mathrm{a}=107.7 / 2 \mathrm{~mm})$, that is, a small antenna. In the best case, how small Q can be? Translating Q in terms of bandwidth, the question reads, in the best condition (one that reaches the $\mathrm{Q}$ limit), how much BW can 
we get from that volume? Is it enough to satisfy the BW requirement for the FM system?

If we assume that the antenna is single resonant, that is, not multi-resonances occur in the FM range, BW can be related to $\mathrm{Q}$ as follows:

$$
B W(S W R \leq S)=\frac{S-1}{\sqrt{S} \cdot Q}
$$

Using Chu's limit including a losses, that is, radiation efficiency, minimum Q within a sphere of radius $k a$ results in:

$$
Q=\eta_{r} \cdot\left(\frac{1}{(k a)^{3}}+\frac{1}{k a}\right) \quad \text { eq.2 }
$$

Where $\eta_{\mathrm{r}}$ is the radiation efficiency (we assume a lossless matching network). Combining eq. 1 and eq. 2 for several values of radiation efficiency and SWR, bandwidth results are shown in Table. 1. To design a FM antenna may be possible if we consider a low efficiency antenna around $1 \%$ with $\mathrm{SWR} \leq 6$ as $\mathrm{BWmax}=26,8 \%>\mathrm{FM}$ bandwidth. Taking into account these values of $\eta_{\mathrm{r}}$ and $\mathrm{SWR}=6$, results in an antenna efficiency of $-23 \mathrm{~dB}$. Considering the antenna as a small dipole, that is, directivity of $2 \mathrm{~dB}$, antenna gain may be around $-20 \mathrm{~dB}$. The question is: is such a low gain enough for FM reception? This question is addressed in the next section.

Table. 1 Maximum bandwidth for several spheres of radius $k a$ as a function of radiation efficiency and SWR

\begin{tabular}{|c|c|c|c|}
\hline$\eta_{\mathrm{r}}[\%]$ & $\mathrm{Q}(\mathrm{ka}=0.11)$ & $\mathrm{BW}(\mathrm{SWR}=3)$ & $\mathrm{BW}(\mathrm{SWR}=6)$ \\
\hline 10 & 76,0 & 1,5 & 2,7 \\
\hline 5 & 38,0 & 3,0 & 5,4 \\
\hline 1 & 7,6 & 15,2 & 26,8 \\
\hline
\end{tabular}

To conclude this section, two experiments are carried out: the first one consist on a straight resonant wire at $100 \mathrm{MHz}$ monopole coplanar to the groundplane of $100 \times 40 \mathrm{~mm}^{2}$, the other is a spiral-type monopole resonating at the same frequency: the antenna occupies a volume of $40 \times 20 \times 5(\mathrm{~h}) \mathrm{mm}^{3}$ and is placed on a groundplane of $80 \times 40 \mathrm{~mm}^{2}$; the total handset dimension is $100 \times 40 \mathrm{~mm}^{2}$. Results are obtained using electromagnetic simulation with IE3D MoM code. The packed wire is longer than $1 \mathrm{~m}$ to resonate at $100 \mathrm{MHz}$ due to coupling effects: total wire is $2262 \mathrm{~mm}$. From Fig. 1 it is observed that the straight monopole presents enough bandwidth whereas the packed monopole is narrowband. Furthermore, computed radiation efficiency for the straight monopole is $92 \%$ while is only $0.04 \%$ ! for the packed monopole. The packed monopole presents low efficiency due to not only its small size but also because it needs almost twice length to resonate due to mutual coupling between arms. Moreover, since the volume for the antenna is small, wire width is $0.25 \mathrm{~mm}$; as a consequence ohmic losses are very high. To overcome this initial problem, next section presents an antenna approach that achieves a much higher efficiency. 


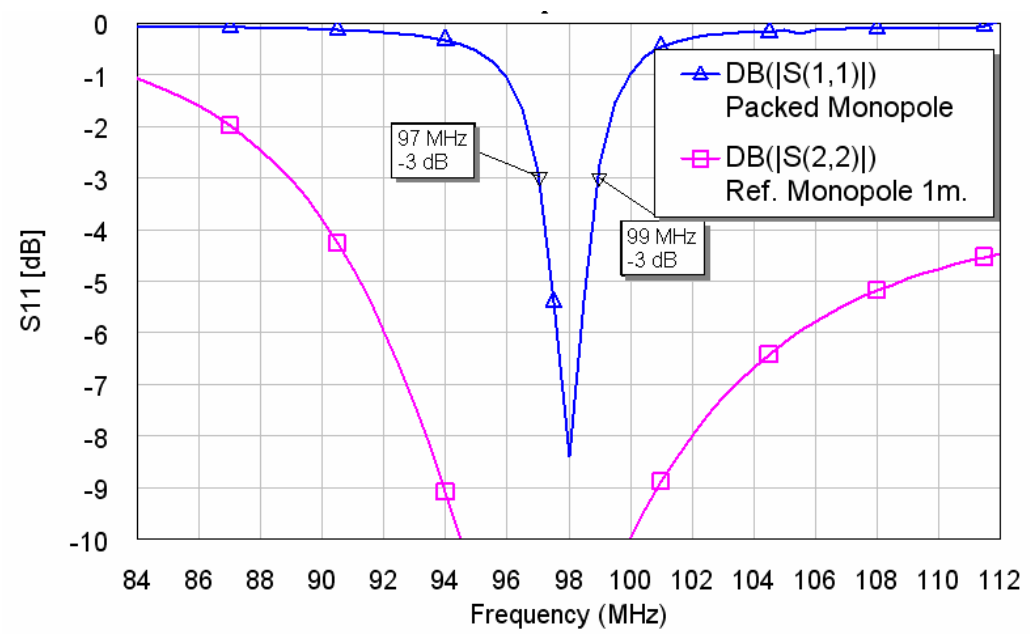

Fig. $1 \mathrm{~S}_{11}$ comparison for the packed and straight monopole (see drawing inTable. 2 )

\section{RESULTS and DISCUSSION}

This section shows an antenna design that achieves a better efficiency and bandwidth that previous case. Antenna uses wider and shorter wire path to minimize ohmic losses. It is tuned to resonance with an external component. The component Q should be taken into account to determine matching losses. For the sake of brevity here, details will be given at the conference.

Three configurations have been simulated, fabricated, and tested. They follow the same antenna concept but they use different groundplane configurations. Each one is representative of different mobile phone platform, namely: a bar-type phone having $100 \times 40 \mathrm{~mm}^{2}$, the same bar-type with $40 \times 20 \mathrm{~mm}^{2}$ clearance for the antenna, and a PDAtype phone having $130 \times 75 \mathrm{~mm}^{2}$. Antennas have been matched with an external inductor (see drawings in Table. 2).

The straight monopole is compared with the packed monopole and the three new antenna proposals [4]. It is clearly shown in Table. 2 how the impedance bandwidths for the new antenna configurations are larger almost reaching the $20 \%$ for $S_{11}<-3 \mathrm{~dB}$ which can be accepted for a reception antenna.

Gain has been measured using a comparison method on an external field. For the three new proposal, gain is around $-20 \mathrm{~dB}$ when the monopole is not above the groundplane. Gain gets worse for the configuration with full-ground. As expected, the monopole near the groundplane presents poor gain, $10 \mathrm{~dB}$ less than previous configurations.

It is interesting to relate results in Table. 1 and Table. 2. For example, prototype one in Table. 2 presents a $k a=0.11$ which is the one used in Table. 1 . If we suppose that directivity is $\mathrm{D}=2 \mathrm{~dB}$ (after simulation), antenna efficiency may be estimated to be $1 \%$ approximately $(-21.1 \mathrm{~dB}$ gain $+2 \mathrm{~dB}$ directivity $=-19.1 \mathrm{~dB}$ antenna efficiency). Table. 2 predicts a maximum allowable bandwidth of $26.8 \%(\mathrm{SWR} \leq 6)$. In this practical case we obtain a bandwidth of $17.8 \%$ which is obviously less than the maximum predicted after Chu's limit.

Finally, to analyze if gain $=-20 \mathrm{~dB}$ is enough for FM reception several experiments have been carried out considering the quality of the demodulated signal. The three configurations have been compared with the straight monopole in term of audio quality not only in free space condition but also in a human body environment. Performance indicates a quality very similar to external monopole. More results will be presented at the conference. 
Table. 2 Measured bandwidth and gain results. Drawing not to scale

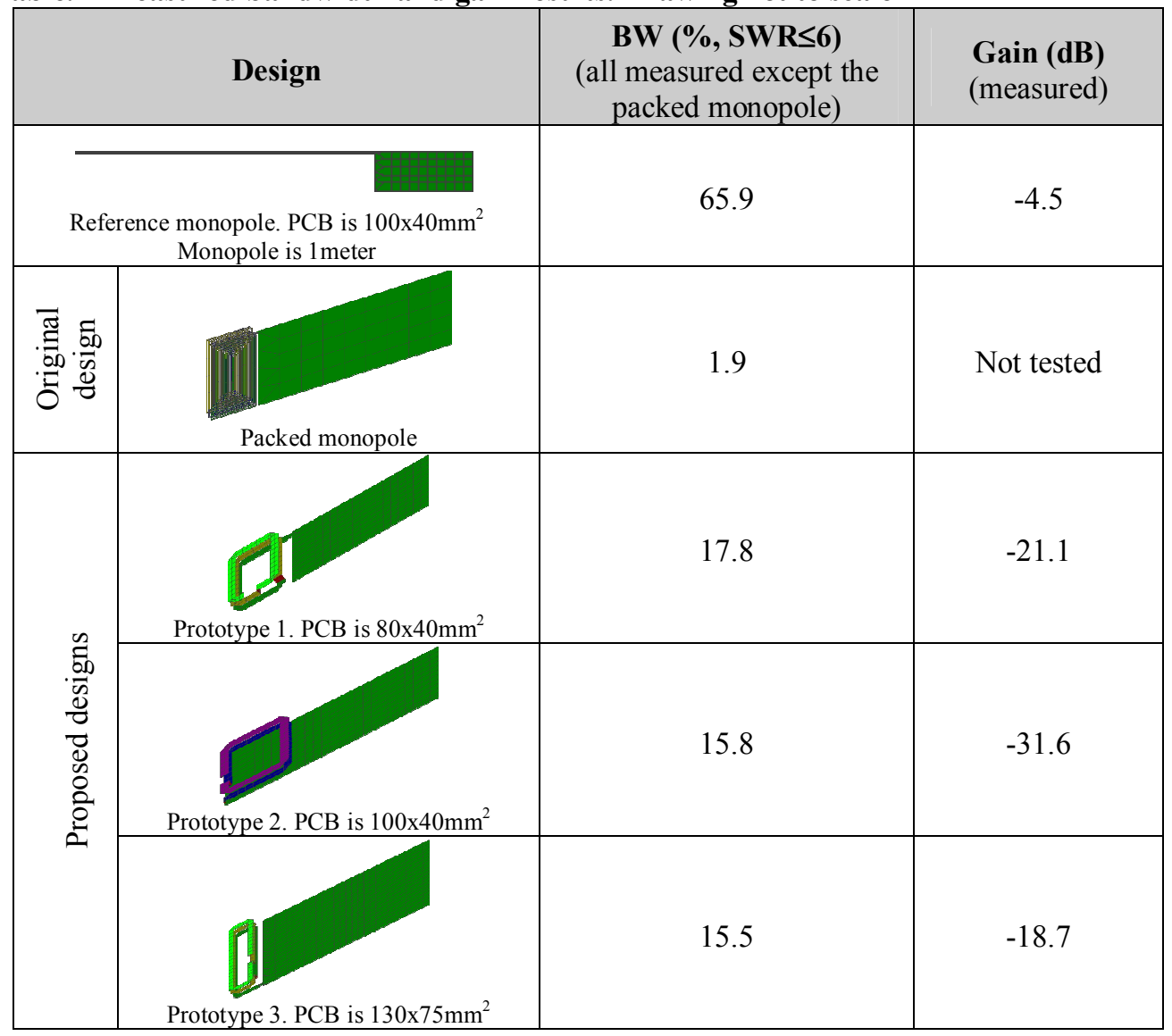

\section{CONCLUSIONS}

Passive internal handset antenna design has been presented. Gain for the tested prototypes is around $-20 \mathrm{~dB}$. Obviously, in weak signal areas, low gain will result in a very poor quality. However, extra research carried out by the authors show that the signal quality with this gain is enough and these prototypes are convenient in a metropolitan area. This research is underway and new results will be presented soon.

\section{ACKNOWLEDGEMENT}

Thanks to the Spanish Ministry of Industry, Commerce, and Tourism for their support to lead to the successful completion of the project.

\section{REFERENCES}

[1] T.Y.Wu and K.L. Wong, "On the impedance bandwidth of a planar inverted-F antenna for mobile handsets", Microwave Opt. Tech. Lett. Vol.32, pp.249-251, Feb.20, 2002

[2] P. Vainikainen, J. Ollikainen, O. Kivekäs, and I. Kelander, "Resonator-based analysis of the combination of mobile handset antenna and chassis," IEEE Transactions on Antennas and Propagation, Vol. 50, No. 10, October 2002, pp. 1433-1444.

[3] J. Anguera, I.Sanz, A.Sanz, A.Condes, D. Gala, C. Puente, and J.Soler, "Enhancing the performance of handset antennas by means of groundplane design". IEEE International Workshop on Antenna Technology: Small Antennas and Novel Metamaterials (iWAT 2006). New York, USA, March 2006.

[4] Patent Pending WO 2007/128340 